TITLE:

\title{
Geometric Invariant theory
}

$\operatorname{AUTHOR}(\mathrm{S})$ :

\section{CITATION:}

Geometric Invariant theory. 数理解析研究所講究録 1981, 444: 38-75

ISSUE DATE:

1981-12

URL:

http://hdl.handle.net/2433/102881

RIGHT: 


\section{Geometric Invariant Theory}

(*) " The story starts with nineteeth century invariant theory, which was concerned with the determination of the invariants of "quantics" (in our language, invariants for the action of $\mathrm{SL}(\mathrm{m}, \mathbb{C}$ ) given by the symmetric power representations). The first step of relevance to us was the proof by Gordon [5] of the finite generation of such invariants in the case $\mathrm{m}=2$, using the methods that had been developed for computational purposes. This result was subsequently extended to arbitrary $m$ by Hilbert [6]; Hilbert's proof depended on his basis theorem (proved in the same paper) and the use of Cayley's $\Omega$-process for constructing a projection from the ring $\mathbf{c}\left[\mathrm{x}_{1}, \ldots, \mathrm{x}_{\mathrm{n}}\right]$ onto the subring of elements invariant under the action of $\mathrm{SL}(\mathrm{m}, \mathbf{C})$.

Various attempts were made in succeeding years to extend Hilbert's result to the natural action of an arbitrary subgroup of $G L(n, \mathbb{C})$ on $\mathbf{c}\left[\mathrm{x}_{1}, \ldots, \mathrm{x}_{\mathrm{n}}\right]$. (See, for example, Hurwitz [9], Fischer [3] for some interesting special cases, and Noether $[12,13]$ for a complete solution in the case of a finite subgroup; this last is valid for any $k$. Unsuccessful attempts on the general problem may be found in Maurer [10,11] and Weitzenbock [14], although the latter contains a correct proof for a subgroup isomorphic to $\mathbf{C}$ or $\mathbf{C}^{*}$ ) (The most significant contribution was that of $\mathrm{H}$. Weyl, whose main results (cf. Weyl [15]) can be stated in our language as follows:

(*) The following is quoted from Newstead [135; 66 of Chap. 3]. We shall make this rather long quotation because this is a good and brief account on the development of the invariant theory. 
(i) any connected semi-simple group over $\mathbf{C}$ is linearly reductive;

(ii) the ring of invariants for a linear action of such a group is finitely generated.

Weyl's original methods combined the integral procedure developed by Hurwitz and Schur and the infinitesimal methods of E. Cartan; subsequently he obtained similar results by algebraic methods, valid for any algebraically closed field of characteristic 0 (cf. Weyl [16]) . In 1933, part of Weyl's argument was simplified and generalized by M. Schiffer, who gave a simple algebraic argument (replacing the use of Cayley's $\Omega$-process) by which (ii) can be deduced from (i) (cf. Weyl [16; 2nd ed. Supplement C]). Schiffer stated his result in a form adapted to the classical case, but his argument is valid generally and indeed in any characteristic; it extends without difficulty to give the following more general result.

THEOREM. For any rational action of a linearly reductive group $G$ on a finitely generated k-algebra $R, R^{G}$ is finitely generated. (A detailed proof of this theorem on the lines of Schiffer's argument may be found in Fogarty [27] or Mumford-Suominen [120] and also, in a slightly disguised form, in Mumford [116]. An alternative proof, due to Nagata, appears in Nagata [64] and Dieudonné-Carrell [25]).

The next advance concerned the construction of counter-examples. In 1900 Hilbert had proposed as his fourteenth problem the following question (cf. Hilbert [8]): 
Is $\mathrm{L} \cap \mathrm{k}\left[\mathrm{x}_{1}, \ldots, \mathrm{x}_{\mathrm{n}}\right]$ a finitely generated $\mathrm{k}$-algebra for every subfield $L$ of $k\left(x_{1}, \ldots, x_{n}\right)$ ?

(Clearly this contains the problem of the finite generation of $k\left[x_{1}, \ldots, x_{n}\right]^{G}$ for a subgroup $G$ of $G L(n)$, though it seems that at the time Hilbert believed that Maurer had solved this problem, at least in the case $\mathrm{k}=\mathbf{C}$.) In 1953 Zariski proposed a generalization of Hilbert's question and answered it in the affirmative in a special case (cf. Zariski [95,96]). However, in 1957, Rees [74] found a counter-example to Zariski's problem; and a year later Nagata [60] gave a negative answer to Hilbert's fourteenth problem itself. In fact, in Nagata's counter-example, $L \cap k\left[x_{1}, \ldots, x_{n}\right]$ is a ring of invariants; so this finally disposed of attempts to prove that such rings are always finitely generated. Over the next few years, Nagata produced a simpler counter-example (cf. Nagata [61]), and also gave a complete identification of linearly reductive groups in arbitrary characteristics (cf. Nagata [62]). (All these results are contained in Nagata's notes on lectures given at the Tata Institute in 1961-62 (cf. Nagata [64]).)"

A modern treatment of the geometric invariant theory perhaps started with Mumford's monograph [116], in which he prefaced that the theory comprises essentially the following two problems:

(i) When does an orbit space of an algebraic scheme acted on by an algebraic group exist?

(ii) Construct moduli schemes for various types of algebraic objects.

Concerning the first problem, he established the concept of orbit 
spaces (or quotient spaces) in explicit and rigorous ways, which had not been sufficiently understood (but only intuitively), and sorted it to give various definitions of quotient spaces, among which the most significant is the concept of geometric quotient. If an algebraic group $G$ acts on an algebraic scheme $\mathrm{X}$, there does not exist in general the geometric quotient of $X$ by $G$ and there arises a question of finding in a natural way a G-invariant open set $U$ of $X$, for which the geometric quotient by $G$ exists; of course, the choice of $U$ has to meet the demands from various moduli problems For this purpose, he introduced the notion of stability (and semistability as well) and proved that the set $x^{\mathbf{S}}$ consisting of all stable points is a G-invariant open set for which the geometric quotient by $G$ exists. Also, he gave an effective criterion to test the stability (also the semi-stability as well) in the case where $G$ is a reductive algebraic group, which is stated in terms of the behaviour of the action of $\mathrm{G}$ on $\mathrm{X}$ when it is restricted to oneparameter subgroups of $G$. In fact, the notion of semi-stability is important to compactify in a natural fashion the moduli scheme obtained as $x^{S} / G$. This theory is tested in various moduli problems, e.g., n-dimensional vector spaces endowed with endomorphisms, binary forms, elliptic curves with neutral points, vector bundles over a curve with given rank and degree, etc.; for the excellent, elementary accounts on these examples, the readers may refer to Mumford-Suominen [121] and Newstead [135]. Mumford applied successfully his theory to the following moduli problems:

(i) $\stackrel{A}{d, g, n}_{\text {: }}$-dimensional abelian varieties with level $\mathrm{n}$ structures and polarizations of degree $d ;$ the fine moduli space 
exists if $n$ is sufficiently large to $g$ and $d$, and the coarse moduli space exists for all possible values of $g, d, n$.

(ii) $\underline{M}_{g}:$ nonsingular curves of genus $g$; the corse moduli space exists.

Mumford's arguments depended greatly on the theory of Hilbert schemes, due to Grothendieck [105], and were restricted to the case of characteristic 0 for the lack of results on the finite generation of the ring of invariants under reductive group actions in the case of positive characteristic. The desired result were conjectured in the preface of [116] as:

MUMFORD'S CONJECTURE. (*) Any reductive algebraic group is geometrically reductive.

The subsequent development of the theory took place surrounding this conjecture. Nagata [65] proved that if a geometrically reductive group $G$ acts rationally on a finitely generated k-algebra $R$ then $R^{G}$ is finitely generated, and Nagata and Miyata [58] proved that a geometrically reductive group is reductive. Oda [68] proved Mumford's conjecture for SL(2) (and hence GL(2)) over a field of characteristic 2 and Seshadri [83] for the same group in general. There is also a contribution by Sumihiro [91]. Finally, in 1974, Haboush [33] proved the conjecture in general, one of the cores in his proof is reduced to the work of Steinberg $[88,89]$. Soon after, Seshadri [86] generalized the results over arbitrary base rings. There are some fundamental works by Seshadri $[84,85]$ on the construction of geometric quotients.

(*) Different in the form from the original one. 
Some positive results have also been obtained for certain actions of non-reductive groups; see, for example, Seshadri [81], Grosshans $[30,31]$ and Hochschild-Mostow [34].

Finally, as introductory books and references, the reporter recommends Seshadri [143], Mumford-Suominen [121], Newstead [135], Fogarty [27], and Dieudonné-Carrell [25] and Springer [*] for classical flavors.

\section{REFERENCES}

(I) Classical invariant theory

For the general list of references, see :

1. Meyer, W.F.: Invariantentheorie. Encyklopädie der mathematischer Wissenschaften, I. B. 2, 1899 .

2. Weitzenböck, R.: Neuere Arbeiten der algebraischen Invariantentheorie. Differentialinvarianten. Encyklopadie der mathematischen Wissenschaften, III. D. 10, 1921.

The other references are:

3. Fischer, E.: Uber algebraische Modulsysteme und lineare homogene partielle Differentialgleichungen mit konstanten Koeffizienten. J. reine angew. Math. 140 (1911), 48-81.

4. Gordan, P.: Vorlesungen uber Invariantentheorie, Bd. 2

(herausgegeben von G. Kerschensteiner). Leibzig, Teubner 1877 .

5. : Beweis dass jede Covariante und Invariante einer

[*] Springer, T.A. : Invariant Theory. Lecture Notes in Math. 585. Springer Verlag, Berlin-Heidelberg-New York, 1977. 
binaren Form eine ganze Funktion mit Coefficienten einer endlicher Anzahl solcher Formen ist. J. reine angew. Math. $69(1868), 323-354$.

6. Hilbert, D.: Über die Theorie der algebraischen Formen. Math. Ann. $36(1890), 473-534$.

7. $42(1893), 313-373$.

8. : Mathematische Probleme. Nachrichten Ges. Wiss. Gottingen (1900), 253-297; Bull. Amer. Math. Soc.8 (1901/2), 437-479 for English translation.

9. Hurwitz, A. : Über die Erzeugung der Invarianten durch Integration. Nachrichten Ges. Wiss. Gottingen (1897), 71-90.

10. Maurer, L. : Über die Endlichkeit der Invariantensysteme. Sitzungsberichte Akad. Wiss. München 29 (1899), 147-175.

11. - Über die Endlichkeit der Invariantensysteme. Math. Ann. 57 (1903), 265-313.

12. Noether, E. : Der Endlichkeitsatz der Invarianten endlicher Gruppen. Math. Ann. 77 (1916), 89-92.

13. - Der Endlichkeitsatz der Invarianten endlicher linearer Gruppen von Charakteristik p. Nachrichten Ges. Wiss. Göttingen (1926), 28-35.

14. Weitzenb8ck, R. : Über die Invarianten von Iinearen Gruppen. Acta Math. 58 (1932), 231-293.

15. Weyl, H.: Theorie der Darstellung kontinuerlicher halb-einfacher Gruppen durch lineare Transformationen. Math. Z. 23 (1925), $271-309 ; 24(1926), 328-395$. 
16. Weyl, H.: The classical groups. Princeton, 1939; 2nd ed., 1946.

17. Elliott, E.B.: An introduction to the algebra of quantics, 2nd ed., Clarendon Press, Oxford, 1913.

18. Grace, J.H. and Young, A.: The algebra of invariants. Cambridge Univ. Press, 1903; Chelsea version is available.

19. Schur, I.: Vorlesungen uber Invariantentheorie. Springer, 1968.

(II) Hilbert's fourteenth problem, Mumford's conjecture and construction of quotient spaces

20. Bia $\chi_{y}$ nicki-Birula, A.: On homogeneous affine spaces of linear algebraic groups. Amer. J. Math. 85 (1963), 577-582.

21 . : Remarks on the action of an algebraic torus on $\mathrm{k}^{\mathrm{n}}$. Bull. de l'Acad. Poln. Sci. I4 (1966), 177-181.

22 . : Some theorems on actions of algebraic groups. Ann.of Math. 98 (1973), 480-497.

23. Birkes, D.: Orbits of linear algebraic groups. Ann. of Math. 93 (1971), 459-475.

24. Cherenack, P.: Submersive and unipotent group quotients among schemes of a countable type over a field k. Trans. Amer. Math. Soc. 211 (1975), 101-112.

25. Dieudonné, J.A. and Carrell, J.B.: Invariant theory, old and new. Academic Press, New York, 1971.

26. Endo, S. and Miyata, T.: Invariants of finite abelian groups. J. Math. Soc. Japan. 25 (1973), 7-26.

27. Fogarty, J.: Invariant theory. Benjamin, New York, 1969.

28. : Fixed point schemes. Amer. J. Math. 95 (1973), $35-51$. 
29. Formanek, E. and Procesi, C.: Mumford's conjecture for the general linear group. Advances in Math. 19 (1976), 292-305.

30. Grosshans, F.: Observable groups and Hilbert's fourteenth problem. Amer. J. Math. 95 (1973), 229-253.

31 . Locarisation and invariant theory. Advances in Math. 21 (1976), 50-60.

32. Gutwirth, A.: The action of an algebraic torus on an affine plane. Trans. Amer. Math. Soc. 105 (1962), 407-414.

33. Haboush, W.J.: Reductive groups are geometrically reductive. Ann. of Math. 102 (1975), 67-83.

34. Hochschild, G. and Mostow, G.D.: Unipotent groups in invariant theory. Proc. Nat. Acad. Sci. U.S.A. 70 (1973), 646-648.

35. Hochster, M. and Fagon, J.A.: Cohen-Macaulay rings, invariant theory and the generic perfection of determinantal loci. Amer. J. Math. 93 (1971), 1020-1058.

36 . and Roberts, J.L.: Rings of invariants of reductive group acting on regular rings are Cohen-Macaulay. Advances in Math. 13 (1974), 115-175.

37. Horrocks, G.: Fixed point schemes of additive group actions. Topology 8 (1969), 233-242.

38. Iversen, B.: A fixed point formula for actions of tori on algebraic varieties. Invent. Math. 16 (1972), 229-236.

39. Kambayashi, T.: Projective representation of algebraic linear groups of transformations. Amer. J. Math. 88 (1966), 199205 .

40 . - Automorphism group of a polynomial ring and algebraic group action on an affine space. J. Algebra 60 (1979), 439-451. 
41. Kambayashi, T., Miyanishi, M. and Takeuchi, M.: Unipotent algebraic groups. Lecture Notes in Math. 414, Springer, 1974.

42. and Russell, P.: On linearizing algebraic torus actions. To appear.

43. Kempf, G., Knudsen, F., Mumford, D. and Saint-Donat, B.: Toroidal embeddings I. Lecture Notes in Math. 339, Springer, 1973.

44. Luna， : Slices étales. Invent. Math.（大南犬追加9项智照)

45. Mabuchi, T.: $\mathbf{c}^{3}$-actions and algebraic threefolds with ample tangent bundle. Nagoya Math. J.

46. : Almost homogeneous torus actions on varieties with ample tangent bundle. Tohoku Math. J.

47. O On the classification of essentially effective $\mathrm{SL}(\mathrm{n}, \mathrm{c})$-actions on algebraic $\mathrm{n}$-folds. Osaka J. Math. 16 (1979), 745-758.

48. : On the classification of essentially effective $\mathrm{SL}(2, \mathbf{C}) \times \mathrm{SL}(2, \mathbb{C})$-actions on algebraic threefolds. Osaka J. Math. 16 (1979), 727-744.

49. : Equivariant embeddings of normal bundles of fixed point loci in varieties under $\mathrm{SL}(\mathrm{m}, \mathbf{C})$-actions. Osaka J. Math. 16 (1979), 707-726.

50. Matsumura, H.: On algebraic groups of birational transformations Rend. Acad. Naz. Lincei 34 (1963), 151-155.

51. Matsushima, Y.: Espaces homogènes de Stein des groupes de Lie complexes. Nagoya Math. J. 16 (1960), 205-218. 
52. Miyake, K. and Oda, T.: Almost homogeneous algebraic varieties under algebraic torus action. Manifolds-Tokyo 1973. Univ. of Tokyo Press, 1975, 373-381.

53. Miyanishi, M.: Some remarks on the actions of the additive group schemes. J. Math. Kyoto Univ. 10 (1970), 189-205.

54. : $\mathrm{G}_{\mathrm{a}}$-actions of the affine plane. Nagoya Math. J. $41(1971), 97-100$

55 On the vanishing of the Demazure cohomologies and the existence of quotient preschemes. J. Math. Kyoto Univ. 11 (1971), 399-414.

56. Introduction à la théorie des sites et son application à la construction des préschémas quotients. Publ. Sém. Math. Sup. 47, Univ. Montréal, 1971.

57. Miyata, T.: Invariants of certain groups. Nagoya Math. J. $41(1971), 69-73$.

58. and Nagata, M.: Note on semi-reductive groups. J. Math. Kyoto Univ. $3(1964), 379-382$.

59. Mumford, D.: Hilbert's fourteenth problem-the finite generation of subrings such as rings of invariants. Mathematical developments arising from Hilbert Problems. Proc. Symp. in Pure Math. 28, Amer. Math. Soc., 1976, 431-444.

60. Nagata, M.: On the fourteenth problem of Hilbert. Proc. Internat. Congress of Math. (Edinburgh 1958), 459-462.

61. : On the fourteenth problem of Hilbert. Amer. J. Math. $81(1959), 766-772$.

62. Complete reducibility of rational representations of a matric group. J. Math. Kyoto Univ. 1 (1961), 87-99. 
63. Nagata, M.: Note on orbit spaces. Osaka Mathi J. 14, 21 (1962) .

64 . Lectures on the fourteenth problem of Hilbert. Tata Institute of Fundamental Research, Bombay, 1965.

65. Invariants of a group in an affine ring. J. Math. Kyoto Univ. $3(1963 / 64), 369-377$.

66. Invariants of a group under a semi-reductive action. J. Math. Kyoto Univ. 5 (1966), 171-176.

67. : Some questions on rational actions of groups. Proc. of the Bombay Colloq. on Algebraic Geometry 1968, Oxford Univ. Press.

68. Oda, T.: On Mumford's conjecture concerning reducible rational representations of algebraic linear groups. J. Math. Kyoto Univ. 3 (1964),

69. : Lectures on torus embeddings and applications. Tata Institute of Fundamental Research. Springer Verlag, BerlinHeidelberg-New York, 1978.

70. Popov, V.L.: Stability criteria for the action of a semisimple group on a factorial manifold. Izv. Akad. Nauk SSSR. Ser. Mat. 34 (1970), 523-531; Math. USSR-Izv. 4 (1970), 527-535.

71. : On stable actions of algebraic groups on algebraic varieties. Izv. Akad. Nauk SSSR. Ser. Mat. 36 (1972), 371385 ; Math. USSR-Izv.

72. - Quasi-homogeneous affine varieties of SL(2). Izv. Akad. Nauk SSSR. Ser. Mat. 37 (1973), 792-832; Math. USSR $-I z v$.

73. and Vinberg, E.B.: On a class of an affine quasihomogeneous space. Izv. Akad. Nauk SSSR. Ser. Mat. 36 (1972) 
749-764; Math. USSR-Izv.

74. Rees, D.: On a problem of Zariski. Illinois J. Math. 2 (1958), 145-149.

75. Rentschler, R.: Opérations du groupe additif sur la plane affine. C. R. Acad. Sci. Paris 267 (1968), A384-A387.

76. Richardson, R.W.: Affine coset spaces of reductive algebraic groups. Bull. London Math. Soc. 9 (1977), 38-4l.

77. Rosenlicht, M.: Toroidal algebraic groups. Proc. Amer. Math. Soc. 12 (1961), 984-988.

78 . A remark on quotient spaces. Annaes Acad. Brasileira de Ciencias 35 (1963), 487-489.

79. Satake, I.: On the arithmetic of tube domains. Bull. Amer. Math. Soc. 79 (1973), 1076-1094.

80. Serre, J.-P.: Groupes finis d'automorphismes d'anneaux locaux réguliers. Colloque d'Algèbre. Secrétariat Math. Paris.

81. Seshadri, C.S.: On a theorem of Weitzenböck in invariant theory. J. Math. Kyoto Univ. 1 (1962), 403-409.

82. Some results on the quotient space by an algebraic group of automorphisms. Math. Ann. 149 (1963), $286-301$.

83 . Mumford's conjecture for GL(2) and applications. Proc. of the Bombay Colloq. on Algebraic Geometry 1968, Oxford Univ. Press, 347-371.

84 . : Quotient spaces modulo reductive algebraic groups and applications to moduli of vector bundles on algebraic curves. Proc. Internat. Congress of Math. (Nice 1970), 
85. Seshadri, C.S.: Quotient spaces modulo reductive algebraic groups. Ann. of Math. 95 (1972), 511-556.

86. : Geometric reductivity over arbitrary base. Advances in Math. 26 (1977), 225-274.

87. Singh, B.: Invariants of finite groups acting on local unique factorization domains. J. Indian Math. Soc. 34 (1970), 31-38.

88. Steinberg, R.: Prime power representations of finite linear groups II. Canad. J. Math. 9 (1957), 347-351.

89. : Representations of algebraic groups. Nagoya Math. J. 22 (1963), 33-56.

90. Shioda, T.: On the graded ring of invariants of binary octavics. Amer. J. Math. 89 (1967), 1022-1046.

91. Sumihiro, H.: Reductive algebraic groups. J. Math. Kyoto Univ. 11 (1971), 527-544.

92. - Equivariant completion I. J. Math. Kyoto Univ. 14 (1974), 1-28; II. 15 (1975), 573-605.

93. Watanabe, K.: Certain invariant subrings are Gorenstein I. Osaka J. Math. 11 (1974), 1-8; II. 11 (1974), 379-388. 94. - Invariant subrings of a Gorenstein local ring by a finite froup generated by pseudo-reflections.

95. Zariski, O.: Interprétations algébro-géométriques du quatorizième problème de Hilbert. Bull. Sci. Math. 78 (1954), 1-14.

96. : The Eheorem of Riemann-Roch for high multiples of an effective divisor on an algebraic surface. Ann. of Math. 76 (1962), 550-615. 
III. Moduli problems and related topics

97. Barth, W.: Moduli of vector bundles on the projective plane. Invent. Math. 42 (1977), 63-91.

98. Desale,U.V. and Ramanan, S.: Poincaré polynomials of the variety of stable bundles. Math. Ann. 216 (1975), 233-244. 99. Classification of vector bundles of rank 2 on hyperelliptic curves. Invent. Math. 38 (1976), $161-185$.

100. Deligne, P. and Mumford, D.: The irreducibility of the space of curves of given genus. Publ. Math. Inst. Hautes Études Sci. 36

101. Dolgachev, I.V. and Ishkovskikh, J.B.: Geometry of algebraic varieties. Algebra, Topology, Geometry. 12 (1974), 77-170; J. Soviet Math.

102. D'Souza, C.: Compactification of generalized Jacobians. Ph. D. Thesis, Univ. of Bombay, 1974 .

103. Gieseker, D.: On the moduli of vector bundles on an algebraic surface. Ann. of Math. 106 (1977), 45-60.

104. : Global moduli for surfaces of general type. Invent. Math.

105. Grothendieck, A.: Fondements de la géométrie algébriqueCollected Bourbaki talks, Extraits du Séminaire Bourbaki, 1957-1962. Paris, 1962.

106. Harder, G.: Eine Bemerkung zu einer Arbeit von P. E. Newstead. J. reine angew. Math. 242 (1970), 16-25.

107. and Narasimhan, M.S.: On the cohomology groups of 
moduli spaces of vector bundles on curves. Math. Ann. 212 (1975), 215-248.

108. Igusa, J.-I.: Fiber systems of Jacobian varieties. Amer. J. Math. 78 (1956), 171-199.

109. Ishida, M.: Compactifications of a family of generalized Jacobian varieties. Proc. Internat. Symp. on Algebraic Geometry Kyoto, 1977.

110. Langton, S.G.: Valuative criteria for families of vector bundles on algebraic surfaces. Ann. of Math. 101 (1975), $88-110$.

111. Maruyama, M.: Stable vector bundles on an algebraic surface. Nagoya Math. J. 58 (1975), 25-68.

112 . Openness of a family of torsion-free sheaves. J. Math. Kyoto Univ. 16 (1976), 627-637.

113. Moduli of stable sheaves. I. J. Math. Kyoto Univ. 17 (1977), 91-126; II.

114. Miranda, R.: On the stability of pencils of cubic curves. To appear.

115. Mumford, D.: Projective invariants of projective structures and applications. Proc. Internat. Congress of Math. ( Stockholm 1962), 526-530.

116. Geometric invariant theory. Springer Verlag, Berlin-Heidelberg-New York, 1965.

117. notes, Wood Hole Summer Institute, 1964.

118. Stability of projective varieties. L'Enseignment Math. 23 (1977), 39- 
119. Mumford, D., Ash, A., Rapoport, M. and Tai, Y.: Smooth compactification of locally symmetric varieties. Math. Sci. Press, 1975.

120 . and Newstead, P.E.: Periods of a moduli space of bundles on curves. Amer. J. Math. 90 (1968), 1200-1208. 121. and Suominen, K.: Introduction to the theory of moduli. Proc. of the fifth Nordic Summer School on Alg. Geometry Oslo, 1970, 171-222.

122. Nakamura, I.: On moduli of stable quasi-abelian varieties. Nagoya Math. J. 58 (1975), 149-214.

123. Namikawa, Y.: A new compactification of the Siegel space and the degeneration of abelian varieties I, II: Math. Ann. 221 (1976), 97-141 and 201-241.

124. Narasimhan, M.S. and Ramanan, S.: Moduli of vector bundles on a compact Riemann surface. Ann. of Math. 89 (1969), 14-51.

125. Vector bundles on curves. Proc. of the Bombay Colloq. on Alg. Geometry. Oxford Univ. Press (1969), 335-346.

126. Deformations of the moduli space of vector bundles over an algebraic curve. Ann. of Math. 101 (1975), 391-417.

127. : Generalized Prym varieties as fixed points. J. Indian Math. Soc. 39 (1975), 1-19. 128. Geometry of Hecke cycles I. 129. and Seshadri, C.S.: Holomorphic vector bundles on a compact Riemann surface. Math. Ann. 155 (1964), 69-80. 
130. Narasimhan, M.S. and Seshadri, C.S.: Stable and unitary vector bundles on a compact Riemann surface. Ann. of Math. 82 (1965), $540-567$.

131. Newstead, P.E.: Topological properties of some spaces of stable bundles. Topology 6 (1967), 241-262.

132 . : Stable bundles of rank 2 and odd degree over a curve of genus 2. Topology 7 (1968), 205-215.

133. : Characteristic classes of stable bundles of rank 2 over an algebraic curve. Trans. Amer. Math. Soc. $169(1972), 337-345$.

134 . - Rationality of moduli spaces of stable bundles. Math. Ann. 215 (1975), 251-268.

135. : Lectures on introduction to moduli problems and orbit spaces. Tata Institute of Fundamental Research, Bombay. Springer Verlag, Berlin-Heidelberg- New York, 1978.

136. Oda, T. and Seshadri, C.S.: Compactifications of generalized Jacobians. Trans. Amer. Math. Soc.

137. Popp, H.: On moduli of algebraic varieties I. Invent. Math. 22 (1973), 1-40; II. Compositio Math. 28 (1974), 51-81; III. Compositio Math. 31 (1975), 237-258.

138. Ramanan, S.: The moduli spaces of vector bundles over an algebraic curve. Math. Ann. 200 (1973), 69-84.

139. Ramanathan, A.: Stable principal bundles on a compact Riemann surface. Math. Ann. 213 (1975), 129-152.

140. Rego, C.J.: The compactified Jacobian. Ann. scient. Ec. Norm. Sup. $13(1980), 211-223$. 
141. Seshadri, C.S.: Space of unitary vector bundles on a compact Riemann surface. Ann. of Math. 85 (1967), 303-336.

142 . : Moduli of $\pi$-vector bundles over an algebraic curve. Questions on algebraic varieties. C.I.M.E., III Ciclo, Varenna 1969, 139-260.

143. : Theory of moduli. Algebraic Geometry Arcata 1974. Proc. Symp. in Pure Math. 29, Amer. Math. Soc. 1975, 263-304 .

144 . Moduli of vector bundles on curves with parabolic structures. Bull. Amer. Math. Soc. 83 (1977), $124-126$.

145 . Desingularization of moduli varieties of vector bundles on curves. Proc. Internat. Symp. on Alg. Geometry Kyoto 1977.

146. Shah, J.: A complete moduli space for K3-surfaces of degree 2 . 147. Takemoto, F.: Stable vector bundles on algebraic surfaces. Nagoya Math. J. 47 (1972), 29-48; II. 52 (1973), 173-195.

148. Tjurin, A.N.: On the classification of two-dimensional vector bundles over an algebraic curve of arbitrary genus. Izv. Akad. Nauk SSSR. Ser. Mat. 28 (1964), 21-52.

149. Classification of vector bundles over an algebraic curve of arbitrary genus. Izv. Akad. Nauk SSSR. Ser. Mat. 29 (1965), 657-688; Amer. Math. Soc. Transl. $63(1967), 245-279$.

150 . : On the classification of $n$-dimensional vector bundles over an algebraic curve of arbitrary genus. Izv. Akad. Nauk SSSR. Ser. Mat. 30 (1966), 1353-1366; Amer. Math. Soc. Transl. 73 (1968), 196-211. 
151. Tjurin, A.N.: Analogue of Torelli's theorem for two-dimensional bundles over algebraic curves of arbitrary genus. Izv. Akad. Nauk SSSR. Ser. Mat. 33 (1969), 1149-1170; Math. USSR-Izv. 3 (1969), 1081-1101.

152 . : Analogues of Torelli's theorem for multidimensional vector bundles over an arbitrary algebraic curve. Izv. Akad. Nauk SSSR. Ser. Mat. 34 (1970), 338-365; Math. USSR-Izv. 4 (1970), 343-370.

153. : The geometry of moduli of vector bundles. Uspehi Mat. Nauk. 29 (1974), 59-88; Russian Math. Surveys $29(1974), 57-88$.

IV. Supplements (not nec. in alphabetical order)

154. Andreev, E.M., Vinberg, E.B. and Elashvili, A.G.: Orbits of greatest dimension in semisimple linear Lie groups. Funct. Anal. appl. 1 (1967), 257-262.

155. Demazure, M.: Démonstration de la conjecture de Mumford (d'après W. Haboush). Sém. Bourbaki Exp. 462. Lecture Notes in Math. 514, Springer, 1976.

156. Dieudonné, J.: La théorie des invariants au XIX ${ }^{e}$ siècle. Sém. Bourbaki Exp.395. Lecture Notes in Math. 244. Springer 1971.

157. Fogarty, J.: Kuhler differentials and Hilbert's fourteenth problem for finite groups. Amer. J. Math. 102 (1980), $1159-1175$.

158. Humphreys, J.E.: Hilbert's fourteenth problem. Amer. Math. Monthly 85 (1978), 341-353. 
159. Luna, D.: Sur les groupes algebriques. Bull. Soc. Math. France Memoire 33, 1973.

160. Morikawa, H.: Theory of invariants. (In Japanese). Kinokuniya, Tokyo, 1977.

161. Nagarajan, K.R.: Groups acting on Noetherian rings. Nieuw Arch. Wisk. (3) 16 (1968), 25-29.

162. Nagata, M. and Otsuka, K.: Some remarks on the 14 th problem of Hilbert. J. Math. Kyoto Univ. 4 (1965), 61-66.

163. Nisnevich, E.A.: Affine homogeneous spaces and finite subgroups of arithmetic groups over function fields. Funct. Anal. Appl. 11 (1977), 64-65.

164. Popov, V.L.: Hilbert's theorem on invariants. Dokl. Akad. Nauk SSSR 249 (1979), 551-554; Soviet Math. Dokl. 20 (1979), $1318-1322$.

165. Rallis, S.J.: New and old results in invariant theory with applications to arithmetic group. Symmetric spaces. Short courses presented at Washignton Univ. Ed. by W.M. Boothley and G.L. Weiss. Pure and applied Math., Marcel Dekker, New York, 1972.

166. Kimura, T. and Sato M.: A classification of irreducible prehomogeneous vector spaces and their relative invariants, Nagoya J. Math., 1976. 
REFERENCES

[1] $\underline{\mathrm{S} . \text { Bloch }}$ : Torsion algebraic cycles, $\mathrm{K}_{2}$ and Brauer groups of function fields, Bull. Amer. Math. Soc. $80(1974), 941-945$.

[2] E. Formanek : The center of the rinf of $3 \times 3$ generic matrices, Lin. Mult. Alg. 7 (1979), $203-212$.

[3] E. Formanek : The center of $4 \times 4$ generic matrices, J.Algebra $62(1980)$, $304-319$.

[4] S. Rosset : Generic matrices, $\mathrm{K}_{2}$, and unirational fields, Bull. Amer. Math. Soc. $81(1975), 707-708$.

[5] L. Snider : Is the Brauer group generated by cyclic algebras? in " Ring Theory, Waterloo, 1978" 279 - 301, LNM 734 (1979).

$-$\begin{tabular}{ll}
000000 & 000000 \\
000000 & 000000 \\
\hline
\end{tabular}

[1] A.A. Albert : Structure of algebras, Amer.Math. Soc. Colloq. Publ. 24.

[2] S.A. Amitsur : On central division algebras, Israel J. Math. 12 (1972), $408-420$.

[3] : The generic divisiob rings, Ibid. 18(1978), $241-247$.

[4 [ and D. Saltman : Generic abelian crossed products and p-algebras, J. Algebra 51(1978), $76-87$.

[5] , L.H. Rowen and J.P. Tignol : Division algebras of degree 4 and 8 with involutions, Israel J. Math. 23(1979), $133-148$.

[6] R. Brauer : On normal division algebras of index 5, Proc. Nat. Ac. Sci. USA. 24 (1958), $243-246$.

[7] C. Chevalley : On algebraic group varieties, J. Math.Soc. Japan 6(1954), $303-324$.

[8] A.L. Cistov : Soviet Math. Dokl. 19(1975), 1267 - 
[9] J.-J. Colliot-Thelene et J. J. Sansuc : La R-equivalence sur les tores, Ann. Sci. Ec. Norm. Sup. $10(1977), 175-230$.

[10] $\underline{\text { S.Endo }}$ and T. Miyata : On a classification of the function fields of algebraic tori, Nagoya Math. J. 56(1974), 85 - 104.

[11] D.R. Farkas : Miscellany on Bieberbach group algebras, Pacific J. Math. $59(\not</ 1975), 427-435$.

[12] K.W. Gruenberg : Relation modules of finite groups, CBMS series 25 (1976)

[13] I.N. Herstein : Notes from a ring conference, Ibid 9 (1971).

[14] N.Jacobson : PI-algebras, An introduction, LNM 441 (1975).

[15] A.Kovacs : Generic splitting fields, Comm. in Algebra 6(1978), 1017 - 1035.

[k6] W. Kuyk : Generic construction of non-cyclic division algebras, J. Pure Appl. Algebra 2(1972), $121-130$.

[17] H.W. Lenstra : Rational functions invariant under a finite abelian group, Inv. Math. $25(1974), 299-325$.

[18] P. Linnel : Zero divisors and idempotents in group rings, Math. Proc. Camb. Phil. Soc. 81(1977), $364-368$.

[19] J.Milnor : Introduction to algebraic K-theory, Ann. Math. Studies 72 (1971)

[20] M. Ojanguren : A remark on generic splitting rings, Arch. der Mat. 27 $(1976), 369-3671$.

[21] F. van Oystaeyen : Generic division algebras, Bull. Soc. Math. Belg. 25 $(1973), 260-285$.

[22] C. Procesi : Noncommutative affine rings, Atti Accad. Naz. Lincei 8(1967), $239-255$.

[23] Rings with polynomial identities, Dekker, New York 1973. [24] The invariant theory of nxn matrices, Adv. in Math. 19 $(1977), 306-381$. 
[25] L. Risman : NOncyclic division algebras, J. Pure Appl. Algebra 11 (1977), $199-216$.

[26] : Cyclic algebras, complete fields, and crossed products, Israel J. Math. 28(1977), $113-128$.

[27] S. Rosset : Abelian splitting fields of division algebras of prime degree, Comment. Math. Helvetici $59(1977), 519-523$.

[28] I.H. Rowen : Central simple algebras, Israel J. Math. $29(1978), 285-301$. : Polynomial identities in ring theory, Academic Press 1980.

[30] : Central simple algebras with involutions, J. Algebra 63 $(1980), 41-55$.

[31] D.Saltman : Splitting of cyclic p-algebras, Proc. Amer. Math. Soc. 62 $(1977), 223-228$.

[32] : Non-crossed products of small exponents, Proc. Amer. Math. Soc. $68(1978), 165-168$.

[33] : Non-crossed product p-algebras and Galois p-extensions,

J. Algebra $52(1978), 526-539$.

[34] R.G. Swan : Invariant rational functions and a problem of Steenrod, Inv. Math. $7(1969), 148-158$.

[35] J. Tignol : Sur les classes de similitude de corps a involution de degree'8, C. R. Acad. Sci. Paris A $286(1978), 375-376$.

[36] V.-E. Voskresenskii : Fields of invariants of abelian groups, Russian Math. Surveys $28(1973), 79-105$.

000 000

[1] D.D. Triantaphyllou : Invariants of finite groups acting non-linearly on rational function fields, J. Pure Appl. Algebra 18(1980), 315 - 331 .

[2] T. Miyata : Invariants of certain groups, Nagoya Math. J. 41(1971), $69-73$. 
(A)

1942 W.V.D. Hodge, A note on k-connexes, Proc. Cambridge Phø̇l. Soc. 38 (1942), 129-143.

1942 D.E. Littlewood, On the number of termes in a simple algebraic form, ibid., 394-395.

1943 W.V.D. Hodge, Some enumerative results in the theory of forme, ibid. $39(1943), 22-30$.

(1927) A. Young, On quantative substitutional analysis (3rd Paper), Proc. London Math. Soc. (2), 28 (1928), 255-292.

$1954 \mathrm{~J}$. Igusa, On the arithmetict normality of the Grassman-variety, Proc. Nat. Acad. Sci. U.S.A. 40 (1954), 309-313.

(B)

1974 P. Doubilet, G.-C. Rota and J. Stein, On the foundations of combinatorial theory:IX- Combinatorial methods in invariant theory, Studies appl. Math. 53 (1974), 185-216.

1976 C. De Contini and C. Procesi, A characteristic free approach to invariant theory, Adv. in Math. 56, $1219330-354$.

1978 ‥ Desarmenien, J.P.S. Kung and G.-C. Rota, Invariant theory, Young bitableaux, and combinatrics, Adv. in Math. 27 (1978), 63-92.

1980 C. De Contini, D. Eisenbud and C. Procesi, Young diagrams and determinalntal varieties, Invent. Math. 56 (1980), 129-165.

1979 C. De Contini, Symplectic standard tableaux, Adv. in Math. 34 (1979), $1-27$.

1979 C. De Contini and E. Strickland, Traceless tensors and the symplectic group, J. of Alg. 61 (1979), 112-128.

1980 A. Abeasis and A. Del Fra, Young diagrams and ideals of Pfaffians, Adv. in Math. 35 (1980), 158-178. 
1980 E. Strickland, The symplectic group and determinants, j. of Alg. $66(1980), 511-533$

1976 P. Doubilet and G.-C. Rota, Skew-symmetric invariant theory, Adv. in Math. 21 (1976), 196-201.

(c)

1971 J. Eagon and M. Hochster, Cohen-Macaulay rings, Invariant theory and the generic perfection of determinantal loci, Amer. J. Math. 93 (1971), 1020-1058.

1972 D. Laksov, The arithmetic Cohen-Macauley character of Schubert schemes, Acta Math. 129 (1972), 1-9.

1972 C. Musili, Postulation formula for Schubert varieties, J. Indian Math. 36 (1972), 143-171.

1973 M. Hochster, Grassmannians and their Schubert subvarieties are arithmetically Cohen-Macauley, J. of Alg. 25 (1973), 40-57.

1979 H. Kraft and C. Procesi, Closur of conjugacy classes of matrices are normal, Inv. Math. 53 (1979), 227-247.

(D) 
Invariant subrings of finite groups which are complete intersections (char. $=0$ ).

*C.Chevalley; Invariants of finite groups generated by reflections, Amer. J. Math. 67 (1955), 778-782.

*M.Hochster and J.Eagon; Cohen-Macaulay rings, invariant theory and the generic perfection of determinantal loci, Amer. J. Math. 93 (1971), 1020-1058.

*K.Watanabe; Certain invariant subrings are Gorenstein, I, II, Osaka J. Math. II (1974), 1-8, 379-388.

*T.A.Springer; Invariant theory; Lect. Notes in Math. 585 Springer, 1977.

*R.Stanley; Relative invariants of finite groups generated by pseudo-reflections, J.Alg. 49 (1977), 134-148.

* ; Invariants of finite groups and their applications to combinatorics, Bull. A.M.S. I (1979), 475-511.

*K.Watanabe; Invariant subrings which are complete intersections, I, (Invariant subrings of finite Abelian groups), Nagoya Math. J. $77(1980), 89-98$.

*__ Invariant subrings of finite groups which are complete intersections, to appear in "Commutative Algebra: Analytic Methods" (Dekker, 1981).

*D.Rotillon; These, Univ. Paris-Nord, 1981.

${ }^{*}$ H.Nakajima; Solution of a problem of Stanley (in Japanese).

*G.C.Shephard and J.A.Todd; Finite reflection groups, Canad. J. Math. 6 (1954), 274-304.

*A.M.Cohen; Finite complex reflection groups, Ann. sc. E.N. S. 9 (1976), 379-436.

*H.F.Blichfeld; Finite collineation groups, Univ. Chicago Press, 1917.

*W.C.Huffman; Linear groups containing an element with an eigenspace of codimension two, J.Alg. 34, 260-287 (1975).

* _ i Imprimitive linear groups generated by elements containing an eigenspace of codimension two, J.Alg. 63 (1980), 499-513.

*M.Schlessinger; Rigidity of quotient singularities, Invent. Math. 14 (1971), 17-26.

*J.Lipman and B.Teissier; On a theorem of Briancon-Skoda about integral closure of ideals, (preprint). 
Algebras with Straightening Laws, Cohen-Macaulay complexes and Cohen-Macaulay Ordered sets.

*D.Eisenbud; Introduction to algebras with straightening laws, Ring theory and alg., III, 1980, 243-268 (Dekker).

*C.De Concini, D.Eisenbud and C.Procesi; Algebras with straightening laws, in preparation.

*R.Stanley; Cohen-Macaulay rings and constructible polytopes, Bull. A.M.S. 81 (1975), 133-135.

*__ The Upper Bound Conjecture and Cohen-Macaulay rings, Studies in Applied Math. 54 (1975), 135-142.

*__ Theory and applications of plane partitions, I,II, Studies in Applied Math., 50 (197I), 167-188, 259-279. *__ S Some combinatorial aspects of the Schubert calculus, in "Combinatoire et Representation du Groupes Symetrique, L.N.Math. 5.79 (1977), 217-251, Springer.

*__ Weyl Groups, the hard Lefschetz theorem and the Sperner property, SIAM J. Alg. Disc. Meth. 1, (1980), 168-184. *__ Unimodal sequences arising from Lie algebras, Proc. Young Day Symp., 127-136, Dekker, 1980. * $\quad$; Hilbert functions of graded algebras, Adv. in Math. $28,57-83$ (1978).

* _ C Cohen-Maculay complexes, Higher Combinatorics (M.Aigner, ed.), Reidel Dordrecht and Boston, Mass., 1977, 51-62. * *G.Reisner; Cohen-Macaulay quotients of polynomial rings, Adv. in Math. 21 (1976), 30-49.

*M.Hochster; Cohen-Macaulay rings, combinatorics and simplicial complexes, Ring Theory, II, 171-223, Dekker, 1977. *K.Baclawski; Cohen-Maculay ordered sets, J. Alg. 63, 226$258(1980)$. * _ Rings with lexicographic straightening law, (preprint) .

* - Cohen-Macaulay connectivity and geometric lattices, (preprint).

*A.Bjorner; Shellable and Cohen-Macaulay partially ordered sets, Trans. A.M.S. $\underline{260}=(1980), 159-183$.

*A.M.Garsia; Combinatorial methods in the theory of CohenMacaulay rings, Adv. in Math. 38 (1980), 229-266.

*A.Bjorner and M.Wachs; Bruhat order of Coxeter groups and shellability, (preprint). 
References for conjugacy classes (incomplete)

[1] P. Bala, R. Carter : Classes of unipotent elements in simple algebraic groups I, Math. Proc. Cambridge Philos. Soc. 79 (1976) 401-425; II, ibid. 80 (1976) 1-17

[2] A. Borel et al.: Seminar in algebraic groups and related finite groups, Springer LNM \#131 1970

[3] W. Borho, H. Kraft : Über Bahnen und deren Deformationen bei linearen Aktionen reduktiver Gruppen, Comment. Math. Helv. 54 (1979) 61-104

[4] J.-L. Brylinski, M. Kashiwara : Kazhdan-Lusztig conjecture and holonomic systems, preprint

[5] E.B. Dynkin : Semisimple subalgebras of semisimple Lie algebras, Amer. Math. Soc. Transl. Ser.2, 6(1957) 111-245

[6] G. Elkington : Centralizers of unipotent elements in semisimple algebraic groups, J. Alg. 23 (1972) 137-163

[7] M. Gerstenhaber : On dominance and varieties of commuting matrices, Ann. of Math. 73 (1961) 324-348 : Dominance over the classical groups, Ann. of Math. 74 (1961) $532-569$

[9] W. Hesselnk : Singularities in the nilpotent scheme of a classical group, Trans. AMS 222 (1976) 1-32

[10] : Closures of orbits in a Lie algebra, Comment. Math. Helv. 54 (1979) 105-110

[11] V. Kac : Some remarks on nilpotent orbits, J. Alg. 64 (1980) 190-213

[12] D. Kazhdan, G. Lusztig : Representations of Coxeter groups and Hecke algebras, Invent. Math. 53 (1979) 165-184 : Schubert varieties and Poincare duality, Proc. AMS Institute "Geometry of Laplacians" 1980

[14] B. Kostant : Lie group representations on polynomial rings, Amer. J. Math. 85 (1963) $327-402$ 
[15] H. Kraft : Parametrisierung von Konjugationsklassen in $s l_{n}$, Math. Ann. 234 (1978) 209-220 : Preprint to appear in Proc. Torun Conference-Asterisque , C. Procesi : Closures of conjugacy classes of matrices are normal, Invent. Math. 53 (1979) 227-247

Univ. Padova, 59(1978) 209-222 : Minimal singularities in $\mathrm{GL}_{\mathrm{n}}$, preprint

[20] G. Lusztig : On the finiteness of the number of unipotent classes, Invent. Math. 34 (1976) 201-213

[2I] K. Mizuno : The conjugate classes of Chevalley groups of type $E_{6}$, J. Fac. Sc. Univ. Tokyo 24 (1977) 525-563

[22] : The conjugate classes of unipotent elements of the Chevalley groups $E_{7}$ and $E_{8}$, Tokyo J. Math. 3 (1980) 391-459

[23] H. Naruse : Classical group aWeyl 群とHecke境 凊现

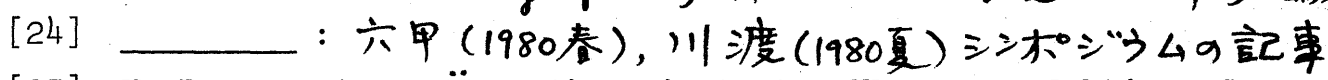

[25] K. Pommerening : Über die unipotenten Klassen reduktiver Gruppen, J. Alg. 49 (1977) 525-536; II, ibid. 65 (1980) 373-398

[26] J. Sekiguchi, Y. Shimizu : Simple singularities and infinitesimally symmetric spaces, Proc. Japan Acad. 57 (1981) 42-46

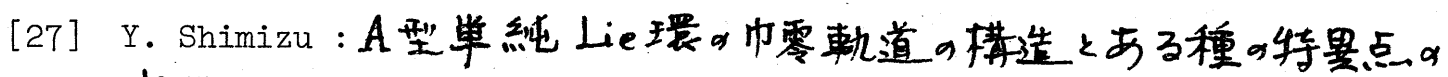
南係にっい2，修土論文，都立大1980（[19]と同样9結果を独立に与えた）

[28] N. Shimomura : A theorem on the fixed point set of a unipotent transformation on the flag manifold, J. Math. Soc. Japan 32 (1980) $55-64$

[29] K. Shinoda : The conjugacy classes of Chevalley groups of type $\left(\mathrm{F}_{4}\right)$ over finite fields of characteristic 2, J. Fac. Sc. Univ. Tokyo 21 (1974) 133-159

[30] T. Shoji : The conjugacy classes of Chevalley groups of type $\left(\mathrm{F}_{4}\right)$ over finite fields of characteristic $p \neq 2$, J. Fac. Sc. Univ. Tokyo 21 (1974) 1-19 
[3I] N. Spaltenstein : The fixed point set of a unipotent transformation on the flag manifold, Proc. Kon. Ak. van Wet. Amsterdam 79 (1976) $452-456$

[32] : On the fixed point set of a unipotent element on the variety of Borel subgroups, Topology 16 (1977) 203-204 : Sous-groupes de Borel contenant un unipotent donné, to appear in Springer LNM

[34] T.A. Springer : The unipotent variety of a semisimple group, Bombay Coll. on Alg. Geom., 373-391, Oxford

[35] B. Srinivasan : Representations of finite Chevalley groups, Springer LNM \#764

[36] R. Steinberg : Classes of elements of semisimple algebraic groups, Proc. Int. Congr. Math. Moscow, (1966) 277-284

[37] : Conjugacy classes in algebraic groups, Springer LNM \#366

[38] : On the desingularization of the unipotent variety, Invent. Math. 36 (1976) 209-224

[39] : Kleinian singularities and unipotent elements, Proc. AMS Institute on finite groups, 1979

[40] G.E. Wall : On the conjugacy classes in the unitary, symplectic and orthogonal groups, J. Austral. Math. Soc. 3 (1963) 1-62 


\section{References for Springer's representations}

[1] R. Hotta, N. Shimomura : The fixed point subvarieties of unipotent transformations on generalized flag varieties and the Green functions, Math. Ann. 241 (1979) 193-208

[2] , T.A. Springer : A specialization theorem for certain Weyl group representations and an application to the Green polynomials of unitary groups, Invent. Math. 41 (1977) 113-127

[3] A. Joseph : Towards the Jantzen conjecture, Compositio Math. 40 (1980) $35-78$

[4] D. Kazhdan, G. Lusztig : A topological approach to Springer's representations, Adv. in Math. 38 (1980) 222-228

[5] G. Lusztig : On the reflection representation of a finite Chevalley group, "Representation theory of Lie groups" ed. by Atiyah, Oxford : Some problems in the representation theory of finite Chevalley groups, Proc. AMS Institute on finite groups, 1979

[7] _ : Green polynomials and singularities of unipotent classes, preprint , N. Spaltenstein : Induced unipotent classes, J. London Math. Soc. 19 (1979) 41-52

[9] T. Murakami：E6 型Weyl group a Springer 表現，千莱大修士諭文(1981)

[10] C. De Concini, C. Procesi : Symmetric functions, conjugacy classes and the flag variety, preprint

[1]] T. Shoji : On the Springer representations of the Weyl groups of classical algebraic groups, Comm. in Alg. 7(16) (1979) 1713-1745; Correction to the above paper, ibid. 7(18) (1979) 2027-2033

[12] _ : On the Springer representations of Chevalley groups of type $F_{4}$, ibid. 8(5) (1980) 409-440

[13] _ On the Green polynomials of a Chevalley group of type $F_{4}$, preprint

[14] P. Slodowy : Simple singularities and simple algebraic groups, Springer LNM \#815

[15] : Four lectures on simple groups and singularities, Comm. Math. Institute Rijksuniversiteit Utrecht, 11-1980

\section{訂正: [10]ค[1]}

\section{$[1] \sim[9] \mapsto[2] \sim[10] 4$}


[16] T.A. Springer : Trigonometric sums, Green functions of finite groups and representations of Weyl groups, Invent. Math. 36 (1976) 173-207

[17] : A construction of representations of Weyl groups, ibid. 44 (1978) 279-293

[18] : Geometric questions arising in the study of unipotent elements, Proc. AMS Institute on finite groups, 1979

兰a他, 岐卓(1977), 札幌(1979)、箱根(1979)、 名古尾(1980) シンポジンムの記事。 
Richard P. Stanley

1. Invariants of finite groups and their applications to combinatorics, Bull. of A.M.S. vol.1, No.3, (1979), 475-511.

2. Hilbert functions of graded algebra, Advances in Math. 28 (1978), 57-83.

3. Relative invariants of finite groups generated by pseudo-reflections, J. Algebra 49 (1977) 134-148.

4. Some combinatorial aspects of the schubert calculus, in "Proc. Table Ronde, Combinatoire et Représentation du Groupe Symétrique, Strasbourg (26-30 Avril 1976), "Lecture note in Math. No.579, pp. 217-25l, Springer, Berlin 1977.

5. The upper bound conjecture and Cohen-Macaulay rings. Stud. in Appl. Math. 54 (1975), 135-142.

6. Cohen-Macaulay rings and constructible polytopes. Bull. Amer. Math. Soc. 81 (1975), 133-135.

7. Combinatorial reciprocity theorem, in "Combinatorics" (M. Hall. Jr. and J.H.Van Lint. Eds.). Part 2. pp.107-118. Mathematical Centre Tracts. No. 56, Mathematisch Centrum, Amsterdam. 1974.

8. Combinatorial reciprocity theorems, Advances in Math. 14 (1974). 194-253.

9. Linear homogeneous diophantine equations and magic labelings of graphs. Duke Math J. 40 (1973). 607-632.

10. Ordered structures and partitions, Mem. Amer. Math. Soc. 119 (1972).

//. Theory and application of plane partitions, Part 1 and Part 2, Stud. in App1. Math. 50 (1971), 167-188, 259-279.

12. Acyclic orientations of graphs, Discrete Math. 5 (1973) 171-178.

13. A Brylawski decomposition for finite ordered sets. Discrete Math. 4 (1973). 77-82.

14. Ordered structures and partitions, Ph. D. dissentation, Harvard University. January. 1971.

15. A chromatic-like polynomial for ordered sets, Proc. Second Chapel Hill Conference on Combinatorial Mathematics and its Applications, (May, 1970) 421-427.

16. Supersolvable semimodular lattices, Algebra Universalis.

17. Fibonacci lattice, Fibonacci Quartely.

18. The conjugate trace and trace of a plane partition, J. Combinatoria] Theory. 
19. Solution to problem 5641 (proposed by Stanley Rabinowitz), Amer. Math. Monthly 76 (1969), 1153.

20. N.J.A. Sloane, Error-Correcting Codes and Invariant Theory: New applications of a nineteenth-century technique, Amer. Math. Monthly 84, 1977, 82-107.

21. N.J.A. Sloane, C.L. Mallows and A.M. Odlyzko, Upper Bounds for Modular Forms, Lattices, and Codes, J. of A1g. 36, $68-76,1975$.

22. Andrew M. Gleason, Weight polynomials of self-dual codes and the Marc Williams identities, Actes, Congres intern. Math. 1970, Tome 3, 211-215.

23. N.J.A. Sloane, Codes over GF(4) and complex lattices, J. algebra $52,168-181,1978$.

24. F.J. Macwilliams, A.M. Odlyzko, N.J.A. Sloane, and H.N. Ward, Self dual codes over GF(4), J. combinatorial theory, Series A25, 288-318, 1978 .

25. F.J. Macwilliams, N.J.A. Sloane, and J.G. Thompson, on the existence of a projective plane of order 10 , J. combinatorial theory, Series A14, 66-78, 1973.

26. F.J. Macwilliams, and N.J.A. Sloane, The theory of errorcorrecting codes, North-Holland, 1978.

27. N.J.A. Sloane, Binary codes, lattices and sphere-packings, Combinatorial surveys: proc. of the sixth British combinatorial conference, 117-164, 1977, Academic press. 
1。森川, 不変式論, 纪国屋。

2. H. Morikawa, Nagoya Math. J. 77 (1980), 75-87.

$3 . \quad$, " 80 (1980), 1-45.

4. R.A. Rankin, J. Indian Math. Soc. 20 (1956), 103-116.

5. E.J. Wilcynski, Projective differential geometry of curves and ruled surfaces (Chelsea).

6. I. Schur, Vorlesungen über Invariantentheorie.

7. H. Morikawa, On Poisson brackets of semi-invariants, 松島教授還曆記念号。 
74

文献透加

Akerman : Hilbert's invariant theory papers (with comments by R.Herman), Math. Sci. Press, 1978.

G. Almkivist and R.Fossum : Decomposition of exterior and symmetric powers of indecomposable $\mathrm{z} / \mathrm{pz}$-modules in characteristic $\mathrm{p}$ and relations to invariants, 1-111, in Sem. d'Algebre Paul Dubreil 1976 - 1977 (30e Annee), LNM 641, 1978.

A. Fauntleroy and A. Magid : Proper G ${ }^{2}$-actions, Duke J. Math. 43(1976),

A. Fauntleroy : On Weizenbock's theorem in positive characteristic, Proc. Amer. Math. Soc. $64(1977)$, : Algebraic and algebro-geometric interpretations of Weizenbock's problem, J. of Algebra 62 (1980), 21 - 38 .

C.S. Fisher : The death of a mathematical theory: A study on the sociology of knowledge, Arch. for the Hist. of exact Sci., 3(1966), 137 159.

G.B. Gurevich : Foundations of the theory of algebraic invariants, P. Noordhoff $\operatorname{LTD}(1964)$.

V.G.Kac, V.I. Popov and E.B. Vinverg : Sur les groupes linéaire algebriques dont l'algèbre de invariants est libre, C.R. Acad. Sc. Paris, 283 $(1976), 875-878$.

G. Kempf : The Hochster-Roberts theorem of invariabt theory, Michigan Math. J. $26(1979), 19-32$.

D. Luna : Slices étales, Bull. Soc. Math. France, Memoire 33(1973), 81-105. : Adherence d'orbite et invariants, Innent. Math. 29(1975), 231 238.

U. Oberst : The use of representations in the invariant theory of not necessarily reductive group, 112 - 127 in Sem. d'Algébre Paul Dubréil 1976 - 1977 (30é Année), LNM 641, 1978. 
V.L. Popov : Representations with a free module of covariants, Functional Anal. Appl. $10(1977), 242-244$.

C. Procesi : Les bases de Hodge dans la theorie des invariants, 128 - 144, in Sem. d'Algébre Paul Dubréil 1977 - 1978, INM 641, 1978.

G.W. Schwarz : Representations of simple Lie groups with regular rings of invariants, Invennt. Math. $49(1978), 167-191$. : Representations of simple Lie groups with a free module of covariants, Invent. Math. $50(1978), 1-12$.

T.A. Springer : on the invariant theory of $\mathrm{SU}_{2}$, Indago Proc. A $83(1980)$, $339-345$.

D.N. Verma : On a classical stability result on invariants of isotypical modules, J. of Algebra $63(1980), 15-40$.

Th. Vust : Foncteurs polynomiaux et théorie des invariants, 330 - 340, in Sem. d'Algèbre Paul Dubréil (32e Année) LNM 795, 1980. : Sur la théorie des invariants des groupes classiques, Ann. Inst. Fourier $26(1976), 1-31$. : Sur la théorie classiques des invariants, Comment. Math, Helvetici $52(1977), 259295$. 